\title{
Habitable 3D Learning Environments for Situated Learning
}

\author{
William H. Bares \\ Luke S. Zettlemoyer \\ James C. Lester \\ (whbares@eos.ncsu.edu) (lszettle@eos.ncsu.edu) (lester@eos.ncsu.edu) \\ Multimedia Laboratory \\ Department of Computer Science \\ North Carolina State University \\ Raleigh, NC 27695-7534 USA
}

\begin{abstract}
The growing emphasis on learner-centered education focuses on intrinsically motivated learning via engaging problem-solving activities. Habitable $3 D$ learning environments, in which learners guide avatars through virtual worlds for role-based problem solving, hold great promise for situated learning. We have been investigating habitable learning environments by iteratively designing, implementing, and evaluating them. In the Situated Avatar-Based Immersive Learning (SAIL) framework for habitable 3D learning environments, learners navigate avatars through virtual worlds as they solve problems by manipulating artifacts. The SAIL framework has been used to implement CPU CITY, a 3D learning environment testbed for the domain of computer architecture. A visually compelling virtual cityscape of computer components, CPU CITY presents learners with goal advertisements that focus their attention on salient problem-solving sub-tasks. The CPU CITY testbed has produced prototypes that have been evaluated. Pilot studies suggest that habitable learning environments offer a promising new paradigm for educational applications.
\end{abstract}

\section{Introduction}

A recent growing emphasis on learner-centered education has yielded a veritable revolution in educational theory and practice. Current theories of learning and pedagogy revolve around constructivism, which emphasizes the learner's knowledge construction process [12]. Perhaps most central among the tenets of constructivism is that learners should be engaged in active exploration, they should be intrinsically motivated, and they should develop an understanding of a domain through challenging and enjoyable problem-solving activities. Considerable work in the ITS community 
has addressed situated learning in microworlds [2, 5, 7, 15], but has only recently begun to explore techniques for situated learning in 3D environments [13].

Engaging 3D learning environments in which learners guide avatars through virtual worlds hold great promise for learner-centered education. By enabling learners to participate in immersive experiences, 3D learning environments could help students develop a deep, experiential understanding of highly complex biological, electronic, or mechanical systems. If 3D learning environments could enable learners to perform tasks typically performed by components of the system considered, qualitative improvements in learning might be possible. For example, learners could study computer architecture in a virtual computer where they might direct data from RAM memory to the hard disk. Properly designed, 3D learning environments that blur the distinction between education and entertainment could produce learning experiences that are intrinsically motivating and solidly grounded in problem solving.

Our laboratory has been investigating this question by iteratively designing, implementing, and evaluating 3D learning environments. Out of this work has emerged the Situated Avatar-Based Immersive Learning (SAIL) framework for habitable 3D learning environments. In habitable learning environments, learners steer avatars through 3D virtual worlds as they manipulate artifacts in the course of problem solving. Environments designed in the SAIL framework facilitate situated learning by providing learners with:

- Habitable virtual worlds: Inviting 3D virtual navigable worlds which represent the intended spatial mental model of the system being studied, house manipulable artifacts whose functionalities mimic those in the system.

- Lifelike avatars: Inhabiting the virtual worlds are learner-guided avatars capable of navigating the environments and manipulating artifacts within them; learners direct their avatar's behaviors.

- Dynamic goal advertisements: By tracking learners' problem-solving activities with a task model, learning environments allow investigative flexibility while at the same time assisting learners by presenting context-sensitive problem-solving goal reminders.

The SAIL framework has been used to implement CPU CiTY (Fig. 1), a 3D learning environment testbed for the domain of computer architecture. CPU CITY provides learners with a virtual computer housing a CPU, RAM, and a hard disk. To develop an understanding of the activities comprising a computation, learners direct an avatar named Whizlo (Fig. 1) to pick up, transport, and insert data packets into registers. The environment presents the learners with goal advertisements that assist them in focusing their attention on the current sub-task. Pilot studies of the CPU CITY testbed indicate that habitable learning environments offer a new paradigm for educational software that can offer significant potential for situated learning. 


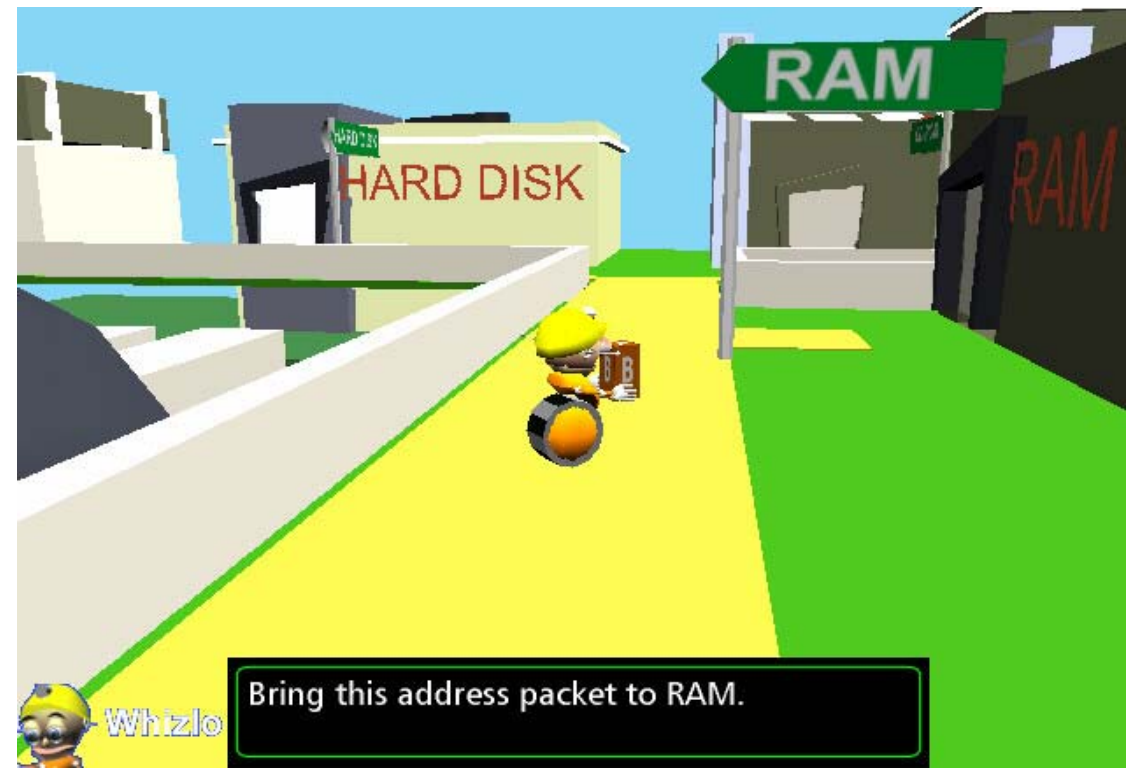

Fig. 1. The CPU CITY Learning Environment

\section{Facilitating Situated Learning}

Over the course of the past few decades, many educators and learning theorists have shifted from an emphasis on rote learning and behaviorist response-strengthening to constructivist learning that emphasizes knowledge construction [10]. In large part because of its emphasis on the learner's active role as he or she acquires new concepts and procedures [12], constructivism has made considerable gains relative to more didactic approaches. A particularly appealing form of constructivist learning is situated learning, which is characterized by intrinsic motivation, situated problem solving, and role-based immersivity.

Intrinsic motivation: Learning should be fun, and a critical feature of many enjoyable experiences is motivation. Two forms of motivation have been identified by cognitive scientists: extrinsically motivating factors, which are external to the learner, e.g. a reward system, and intrinsically motivating factors, which come from within and are usually characterized by playful learning. In the classic study of intrinsic motivation in computer games [9], it was found that (1) three key components of intrinsic motivation are challenge, fantasy, and curiosity, and (2) regarding curiosity, both sensory and cognitive factors are critical. 
Situated problem solving: Previous learning theories tended to emphasize rote memorization. In contrast, situated learning involves situated problem-solving episodes [14]: these goal-directed activities take place in concrete contexts in which learning occurs as a by-product of solving problems. For example, rather than learning algebra through abstract symbol manipulation, learners could design a space station, requiring them to formulate and solve equations.

Role-based immersivity: Recent years have seen a growing interest in story-telling software that enables users to design stories, create storytellers, and actively participate in plots, perhaps taking on the role of one or more of the characters $[4,16]$. A hallmark of situated learning is that the learner takes on an active role in a virtual world that simulates the system being studied. For example, when learning about a computer's fetch-execute cycle, learners could become the machine by taking on the role of a courier whose task is to gather data values, perform computations, and store the results in RAM. The central features of situated learning point towards a new form of educational software that could provide intrinsic motivation, support situated problem solving, and facilitate role-based immersivity.

\section{Situated Avatar-Based Immersive Learning Environments}

To create a new paradigm for educational software that can foster situated learning, we have been iteratively designing learning environment technologies whose evaluations suggest that they offer intrinsic motivation, situated problem solving, and role-based immersivity. Out of this work has emerged the Situated Avatar-Based Immersive Learning (SAIL) framework for habitable 3D learning environments.

For example, in a computer architecture learning environment, they might be asked to guide the avatar as it fetches data from RAM and performs calculations in the CPU. A learner's joystick inputs are interpreted by a world monitor which passes them to an avatar director, a point-of-view composer, and a task modeler (Fig. 2). Learners issue navigation directives and manipulation directives to the avatar director. For example, in the course of solving problems, the learner might direct the avatar to pick up an object, carry an object from one location to another, or insert an object into a receptacle. The task modeler tracks the learner's current goal and calls on the pointof-view composer and cinematography system to compose camera shots to view the avatar and salient objects of the current goal in a way that they are clearly visible [1]. The task modeler uses goal advertisements to give reminders about the purpose of the current sub-task, as well as offering advice about how it should be carried out.

Observations of learners interacting with these environments indicate that three families of design decisions are critical: designing the components and layout of the virtual worlds to create engaging universes that maximize clarity, consistency, and pedagogical value; designing avatars that are lifelike; and designing goal advertisements and a mechanism for presenting them. 


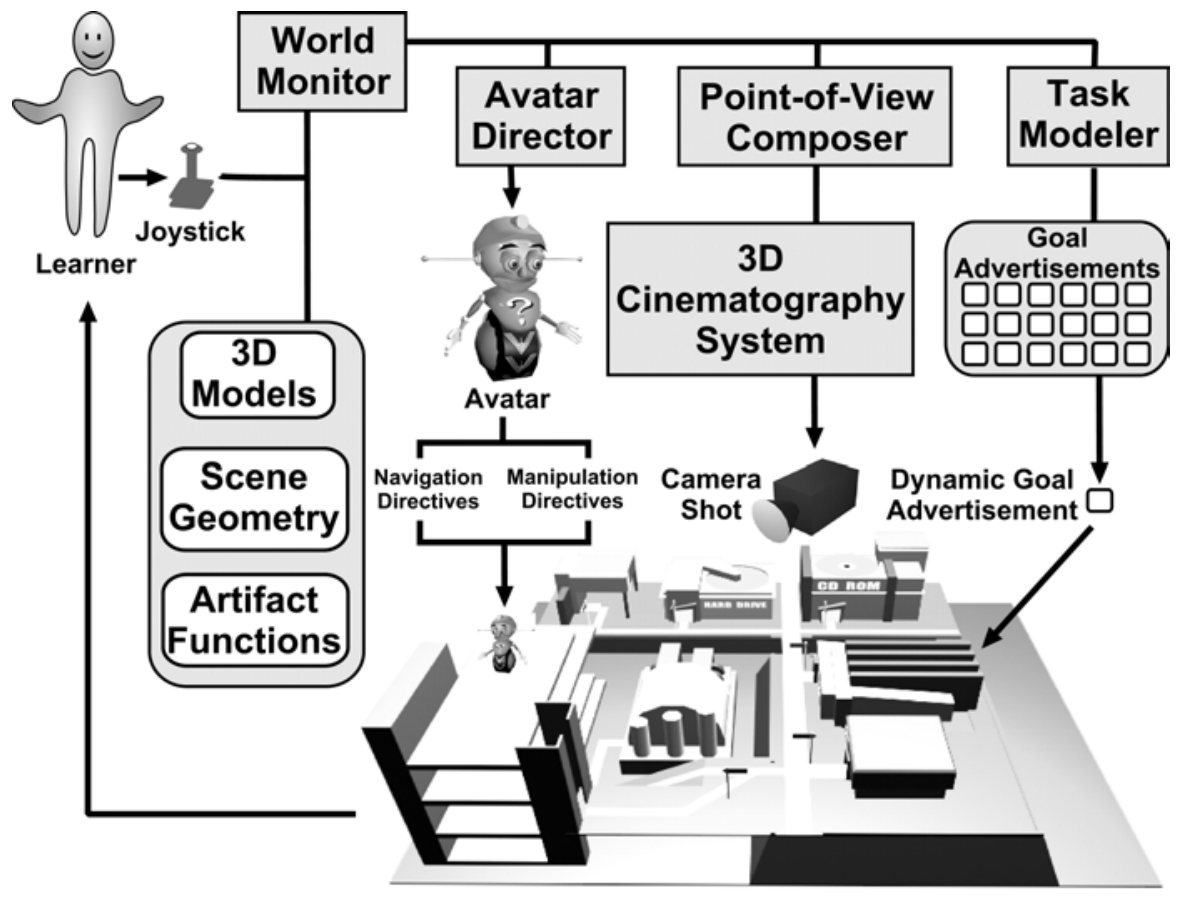

Fig. 2. The SAIL Architecture

\subsection{Habitable Virtual Worlds}

A 3D learning environment's virtual world must define an "information landscape" [3] that clearly communicates the central features of the system to be investigated. To help learners acquire a spatial mental model that reflects reality and a process-based mental model of its functionalities, virtual worlds can be constructed with the following techniques:

Architectural Abstraction: By creating virtual worlds around metaphors that communicate the essential structures and functions of the system, designers can facilitate situated learning. They can provide visual mnemonic devices where important spatial relationships between components are suggested by their layout, and redundant cues such as appearance and labeling assist in their identification. For example, the CPU CITY learning environment testbed is a stylized rendition of an 
actual computer cast as a colorful cityscape. The memory and I/O buses on which data travel are roadways and road signs identify relevant locations.

Spatial and Temporal Scaling: Virtual worlds can take advantage of an animator's ability to scale space and time. By increasing or decreasing the spatial scale, architectural structures can be designed to help learners' acquire a spatial mental model that is on a human scale, even if the physical system being studied is not. For example, the spatial scale of the CPU CITY virtual world was expanded to enable learners to navigate components of approximately human size. Temporal scaling can be equally effective for creating experiences that occur on a human time scale. Through temporal dilation, computations requiring only milliseconds are stretched out to minutes in CPU CITY to provide time for learners to reflect on them.

\section{2 Lifelike Avatars}

Because direct experience seems to act as an exceptionally strong catalyst for learning, providing a learner with an avatar - a physical embodiment of the learner in the 3D world - appears promising from an educational perspective. In addition to the direct pedagogical benefits, lifelike avatars offer significant motivational promise. For much the same reasons that lifelike animated pedagogical agents have proven so successful [8], lifelike avatars seem to emotionally engage the learners who direct them. To achieve this effect, designers can create avatars that are visually compelling by exploiting the behavior cannon of animation [6]. For example, Whizlo (Figure 1), an eager, bright-eyed robot, was given a unicycle-like wheel to scoot through CPU CIтY. While idle he is kept alive with a slight rocking motion as he awaits the learner's next directive.

\subsection{Dynamic Goal Advertisements}

SAIL environments are intended to foster exploratory freedom, one of the hallmarks of situated learning, by allowing learners to explore the virtual world at will. Increased freedom leads to the design challenge of assisting learners to cope with this flexibility by modeling their problem-solving activities and offering guidance. Ideally operating in a guided discovery mode, the environments should enable learners to pursue paths in a curiosity-driven fashion while at the same time providing support. Achieving this delicate balance entails addressing two issues: first, how to dynamically model learners problem-solving activities, and second, how to present support in a contextually appropriate manner.

SAIL environments address the first issue by maintaining a procedural task model and traversing it as learners perform their tasks. In contrast to more complex approaches to task modeling such as task-action grammars (TAGs) [11], procedural task models are comparatively simple. They represent tasks as a network of goals and 
the temporal constraints between them. Each goal has transition condition(s), which when satisfied, lead to a successor goal. For example, in CPU CITY, the goal to pick up an object is satisfied when the learner directs the avatar near the object and picks it up by pushing the joystick action button. While task networks lack the expressiveness and flexibility of elaborate TAGS, they are considerably easier to construct and have proven effective for determining when and which goal advertisements to present for procedural tasks with well-ordered goals.

SAIL environments address the issue of presenting context-sensitive support by adopting a multi-tiered goal advertisement approach. Experience with learners interacting with CPU CITY suggest that at least two forms of assistance are useful:

- Conceptual goal advertisements: One of the most important types of support provided by SAIL environments is helping learners bridge the gap between highlevel concepts and the immediate sub-task being performed. For example, one of the conceptual goal advertisements presented as learners interact with CPU CITY suggests, "Deposit address of A: Place the address of A into the address input port. RAM will then immediately return the numeric value of A. You can think of the address of A like the library call number for a book. The value of A is like the book shelved at that spot in the library."

- Operational goal advertisements: To help learners keep the immediate problemsolving goal in focus, operational goal advertisements present explicit, typically abbreviated, suggestions about the course of action to take. For example, one of CPU CITY's operational goal advertisements suggests, "Walk over to control unit and pick up the address of B packet."

SAIL systems use the current state of the traversal of the task model to index into a library of goal advertisements that are linked by intentional indices to the procedural task network. When a new goal is activated, the relevant conceptual goal advertisement is presented by both text and digitized speech. After the learner clicks the action button to acknowledge that he or she has assimilated the message, the advertisement is removed. Then a corresponding operational advertisement appears at the bottom of the screen to offer a concise reminder about the current goal.

\section{The CPU City Testbed}

The SAIL framework for habitable learning environments has been implemented in CPU CITY, a testbed for the domain of computer architecture. CPU CITY is implemented in 55,000 lines of $\mathrm{C}++$ and uses OpenGL for 3D rendering. Its task model consists of 26 goal states, its goal advertisement library contains over 40 advertisements, and its virtual world houses more than 50 3D models. A fundamental design decision was to target cost-effective platforms likely to be available in the classroom in the near future. To this end, the CPU CITY environment currently runs on a $333 \mathrm{MHz}$ Pentium II with a Permedia2 3D graphics accelerator and $128 \mathrm{MB}$ 
memory at 15 frames/second in a 640x480 full-screen window. CPU CITY provides learners with joystick control, which they use to steer Whizlo through CPU CITY and pickup and deposit objects being carried.

Consider the following example from an ongoing learning session in which the learner has just deposited the value of the first operand, A, whose value is 250 , into CPU register \#1 to continue the process of computing the subtraction of B from A. Verbal praise is given by Whizlo to applaud the student for having achieved this goal. The learner is reminded that in order to fetch the second operand, B, he/she must first obtain the address of that value from the CPU control unit. The learner scoots Whizlo to the control unit and grabs the address of B packet. Next, the learner directs Whizlo to exit the CPU, scurry along the CPU-memory bus pathway to enter the RAM, and positions him in front of the RAM address input port. After hitting the action button to deposit the address packet, the learner is told with a goal advertisement that the value of the second number has been swapped-out to disk since it had not been recently used. Therefore, the learner must retrieve the address packet from the RAM input port and transport it to the hard disk where the value of B can be found in virtual memory. There the learner must obtain the value of $\mathrm{B}$, return it to the $\mathrm{CPU}$ for computation, and lastly, store the result value C into RAM.

\section{Pilot Studies and Design Implications}

To investigate learners' interactions with habitable learning environments, we have conducted a series of informal pilot studies with more than 30 subjects ranging in age from 11 to 53. Approximately one-third of the subjects were female and two-thirds were male. Objectives were to evaluate learner response to immersive problem solving by directing an avatar from the third person point of view with the assistance of task-sensitive goal advertisements. Four conclusions have emerged:

1. Habitable learning environments offer significant promise for true situated learning. Observing learners of different ages interact with CPU CITY suggests that the combination of learner-directed lifelike avatars, engaging 3D virtual worlds, and immersive role-based problem solving has significant potential.

2. Virtual worlds should be inviting and their structure and function should mirror the system being studied at a "learnable" level of abstraction. The architectural components of virtual worlds should mirror their real-world counterparts' structure and functionalities with pedagogically appropriate abstractions. Taking advantage of straightforward metaphors and exploiting time and space scaling may benefit learners considerably.

3. Avatars should be visually compelling, provide manipulative functionalities, and exhibit personality. Lifelike characters that can interact in interesting ways with the environment (more so than our prototype learning environments have 
provided to date) and can perform actions that intrigue the learner may play a central motivational role.

4. Goal advertisements - both conceptual and operational — are critical for effective learning. The immersive effects of a well-designed role-playing task in a compelling environment are very strong. In the absence of goal advertisements, learners may become so engrossed that they may fixate on the immediate sub-task and fail to make conceptual connections. Goal advertisements are one means for bridging the critical gap from concepts to the specific tasks the learner performs.

\section{Conclusions}

Habitable learning environments offer significant potential for fostering true situated learning. By directing lifelike avatars to perform problem-solving tasks in intriguing 3D virtual worlds, learners can be intrinsically motivated, perform situated problem solving, and be actively immersed in a role-based learning experience. By leveraging powerful graphics technologies, learners can interact with what may become a new paradigm for education software in the not too distant future. This work represents a step towards learning environments that support situated learning. It raises many more questions than it answers about learning and the role that technologies can play in nurturing it. We look forward to addressing these issues in our future work.

\section{Acknowledgements}

Thanks to: the design team (Tim Buie, Mike Cuales, and Rob Gray), led by Patrick FitzGerald, for the modeling of the CPU CITY testbed; Francis Fong, for his work on the animation tools; Dennis Rodriguez and Joël Grégoire for their work on the task modeling; and Charles Callaway and Stuart Towns, for comments on earlier drafts of this paper. Support for this work is provided by a grant from the National Science Foundation (Faculty Early Career Development Award IRI-9701503), the North Carolina State University IntelliMedia Initiative, and an industrial gift from Novell.

\section{References}

1. Bares, W.H., Zettlemoyer, L.S., Rodriguez, D.W., and Lester, J.C. TaskSensitive Cinematography Interfaces for Interactive 3D Learning Environments. In Proceedings of IUI '98, San Francisco CA (1998) 81-88

2. Burton, R.R. and Brown, J.S. An Investigation of Computer Coaching for Informal Learning Activities. In D. Sleeman and J.S. Brown, editors, Intelligent Tutoring Systems. Academic Press (1982) 79-98 
3. Davenport, G. Seeking Dynamic, Adaptive Story Environments. In IEEE Multimedia (Fall 1994) 9-13

4. Hayes-Roth, B., and Van Gent, R. Story-Making with Improvisational Puppets. In Proceedings of the First International Conference on Autonomous Agents, Los Angeles CA (1997) 1-7

5. Hollan, J.D., Hutchins, E.L., and Weitzman, L.M. STEAMER: An Interactive Inspectable, Simulation-Based Training System. In G. Kearsley, editor, Artificial Intelligence and Instruction: Applictions and Methods. Addison-Wesley (1987) 113-134

6. Jones, C. Chuck Amuck: The Life and Times of an Animated Cartoonist. Avon, New York NY (1989)

7. Lawler, R.W. and Lawler, G.P. Computer Microworlds and Reading: An Analysis for their Systematic Application. In R.W. Lawler and M. Yazdani, editors, Artificial Intelligence and Education. Ablex (1987) 95-115

8. Lester, J.C., Converse, S.E., Kahler, S.E., Barlow, S.T., Stone, B.A., and Bhogal, R. The Persona Effect: Affective Impact of Animated Pedagogical Agents. In Proceedings of CHI '97 (1997) 359-366

9. Malone, T.W. Toward a Theory of Intrinsically Motivating Instruction. In Cognitive Science 4 (1981) 333-369

10. Mayer, R.E. Educational Psychology: A Cognitive Approach. Harper Collins, New York NY, 1987.

11. Payne, S. J., and Green, T.R. Task-action Grammars- A Model of the Mental Representation of Task Languages. In Human-Computer Interaction 2 (1986) 99-133

12. Piaget, J. The Construction of Reality in the Child. Basic Books, New York NY (1954)

13. Rickel, J. and Johnson L. Intelligent Tutoring in Virtual Reality: A Preliminary Report. In Proceedings of the Eight World Conference on AI in Education (1997) 294-301

14. Suchman, L. Plans and Situated Actions: The Problem of Human Machine Communication. Cambridge University Press, Cambridge MA (1987)

15. Thompson, P.W. Mathematical Microworlds and Intelligent Computer-Assisted Instruction. In G. Kearsley, editor, Artificial Intelligence and Instruction: Applications and Methods. Addison-Wesley (1987) 83-109

16. Umaschi, M. Soft Toys with Computer Hearts: Building Personal Storytelling Environments. In CHI '97 Extended Abstracts, ACM Press (1997) 20-21 